\title{
COMMUTATIVITY AND HYPONORMALITY OF TOEPLITZ OPERATORS ON THE WEIGHTED BERGMAN SPACE
}

\author{
Yufeng Lu And ChaOmei LiU
}

\begin{abstract}
In this paper we give necessary and sufficient conditions that two Toeplitz operators with monomial symbols acting on the weighted Bergman space commute. We also present necessary and sufficient conditions for the hyponormality of Toeplitz operators with some special symbols on the weighted Bergman space. All the results are stated in terms of the Mellin transform of the symbol.
\end{abstract}

\section{Preliminaries}

Let $\mathbb{D}$ be the open unit disk in $\mathbb{C}$, and let $d A$ be the normalized area measure on $\mathbb{D}$. For $-1<\alpha<+\infty$, the weighted Bergman space $A_{\alpha}^{2}$ of the disk is the space of analytic functions in $L^{2}\left(\mathbb{D}, d A_{\alpha}\right)$, where

$$
d A_{\alpha}(z)=(\alpha+1)\left(1-|z|^{2}\right)^{\alpha} d A(z) .
$$

It is well known that $A_{\alpha}^{2}$ is a closed subspace of the Hilbert space $L^{2}\left(\mathbb{D}, d A_{\alpha}\right)$. Let $P$ denote the orthogonal projection from $L^{2}\left(\mathbb{D}, d A_{\alpha}\right)$ onto $A_{\alpha}^{2}$. Let $L^{\infty}(\mathbb{D})$ be the space of essentially bounded area measurable functions on $\mathbb{D}$. For $\varphi \in$ $L^{\infty}(\mathbb{D})$, the Toeplitz operator with the symbol $\varphi$ is the operator $T_{\varphi}: A_{\alpha}^{2} \rightarrow A_{\alpha}^{2}$ defined by

$$
T_{\varphi} f=P(\varphi f), f \in A_{\alpha}^{2} .
$$

This paper is motivated by the following two problems: The first one is to find necessary and sufficient conditions that two Toeplitz operators acting on the weighted Bergman space commute; the second one is to describe hyponormality of Toeplitz operators acting on the weighted Bergman space. Knowing commutativity of two Toeplitz operators and hyponormality of Toeplitz operators often helps us give an idea of what these operators look like; conversely, trying to determine commutativity of two Toeplitz operators and hyponormality of Toeplitz operators often leads to interesting problems in analysis.

Received September 17, 2007.

2000 Mathematics Subject Classification. Primary 47B35, 47B20.

Key words and phrases. weighted Bergman space, Toeplitz operator, Mellin transform, commutativity, hyponormality.

This research is supported by NSFC, Item Number:10671028. 
In the case of the classical Hardy space $H^{2}$, Brown and Halmos [3] characterized commutativity of Toeplitz operators on $H^{2}$. On the Bergman space of the unit disk, the first complete result was obtained by Axler and Čučković who characterized commuting Toeplitz operators with harmonic symbols [1]. Stroethoff extended their result to essentially commuting Toeplitz operators [23]. Axler, Čučković and Rao showed that if two Toeplitz operators commute and the symbol of one of them is analytic and nonconstant, then the other one is also analytic [2]. Čučković, and Rao studied Toeplitz operators that commute with Toeplitz operators with monomial symbols [8]. On the Bergman space of several complex variables, the situation is much more complicated. Zheng studied commuting Toeplitz operators with pluriharmonic symbols on the unit ball in $\mathbb{C}^{n}[25]$; Lee studied weighted cases [17]. Lu characterized commuting Toeplitz operators on the Bergman space of the bidisk with $H^{\infty}\left(D^{2}\right)+\overline{H^{\infty}\left(D^{2}\right)}$ symbols [19]. B. R. Choe, H. Koo, and Y. J. Lee obtained characterizations of (essentially) commuting Toeplitz operators with pluriharmonic symbols on the Bergman space of the polydisk [4].

A bounded linear operator $A$ on a Hilbert space is said to be hyponormal if its selfcommutator $\left[A^{*}, A\right]:=A^{*} A-A A^{*}$ is positive semidefinite. The hyponormality of Toeplitz operators on the Hardy space has been studied by many mathematicians, see $[6,7,9,10,11,12,13,15,16,20,22,26]$. The hyponormality of Toeplitz operators on the Bergman space has been studied by H. Sadraoui [21] and I. S. Hwang [14].

In this paper we find necessary and sufficient conditions for a symbol that produces a Toeplitz operator on the weighted Bergman space that commutes with another such operator whose symbol is a monomial. We also discuss the hyponormality of Toeplitz operators on the weighted Bergman space and give necessary and sufficient conditions for some special symbols that produce hyponormal Toeplitz operators on the weighted Bergman space.

\section{Commutativity of Toeplitz operators}

We start this section with a decomposition of the space $L^{2}\left(\mathbb{D}, d A_{\alpha}\right)$, where $\alpha>-1$. Let

$$
\mathscr{R}_{\alpha}=\left\{a: \mathbb{D} \rightarrow \mathbb{C} \text { radial }\left.\left|\int_{0}^{1}\right| a(r)\right|^{2} r\left(1-r^{2}\right)^{\alpha} d r<\infty\right\},
$$

and let $\mathscr{R}_{\alpha, k}=e^{i k \theta} \mathscr{R}_{\alpha}$ for $k \in \mathbb{Z}$. Since for $u \in \mathscr{R}_{\alpha, k}, u\left(r e^{i \theta}\right)=e^{i k \theta} a(r)$ and

$$
\int_{\mathbb{D}}|u(z)|^{2} d A_{\alpha}(z)=\frac{(\alpha+1)}{\pi} \int_{0}^{1} \int_{0}^{2 \pi}|a(r)|^{2} r\left(1-r^{2}\right)^{\alpha} d \theta d r<\infty,
$$

each $\mathscr{R}_{\alpha, k}$ is a subspace of $L^{2}\left(\mathbb{D}, d A_{\alpha}\right)$. 
It is also clear that $\mathscr{R}_{\alpha, k} \perp \mathscr{R}_{\alpha, l}$ if $k \neq l$. Every polynomial in $z$ and $\bar{z}$ can be written as

$$
p(z, \bar{z})=\sum_{k=-N}^{N}\left(\sum_{i-j=k} a_{i j} z^{i} \bar{z}^{j}\right)
$$

for some $N$, and in polar coordinates it has the form

$$
\sum_{k=-N}^{N} e^{i k \theta}\left(\sum_{i-j=k} a_{i j} r^{i+j}\right) \in \bigoplus_{k \in \mathbb{Z}} e^{i k \theta} \mathscr{R}_{\alpha} .
$$

Since the set of all the polynomials $p(z, \bar{z})$ is dense in $C(\overline{\mathbb{D}})$ and $C(\overline{\mathbb{D}})$ is dense in $L^{2}\left(\mathbb{D}, d A_{\alpha}\right)$, we can conclude that $L^{2}\left(\mathbb{D}, d A_{\alpha}\right)=\bigoplus_{k \in \mathbb{Z}} e^{i k \theta} \mathscr{R}_{\alpha}$, i.e., every function $u \in L^{2}\left(\mathbb{D}, d A_{\alpha}\right)$ has the form

$$
u\left(r e^{i \theta}\right)=\sum_{k=-\infty}^{\infty} e^{i k \theta} u_{k}(r), \quad u_{k} \in \mathscr{R}_{\alpha} .
$$

One of our most useful tools in the following calculations is the Mellin transform (closely related, using the change of variables $x=e^{-u}$, to the Laplace transform). The Mellin transform $\widehat{\varphi}$ of a function $\varphi$ is defined by the equation

$$
\widehat{\varphi}(z)=\int_{0}^{\infty} \varphi(x) x^{z-1} d x .
$$

By [18] we know that $\widehat{\varphi}(z)$ is well defined on $\{z \in \mathbb{C}: \operatorname{Re} z \geq 2\}$ and analytic on $\{z \in \mathbb{C}: \operatorname{Re} z>2\}$ if $\varphi \in L^{1}([0,1], r d r)$.

Now we start to discuss the commuting problem of two Toeplitz operators on the weighted Bergman space. Let $\mathbb{N}_{0}=\mathbb{N} \bigcup\{0\}$. While our method is partially adapted from [8], substantial amount of extra work is necessary for the setting of the weighted Bergman space.

Theorem 2.1. Let $\psi\left(r e^{i \theta}\right)=\sum_{k=-\infty}^{\infty} e^{i k \theta} \psi_{k}(r)$ and $\varphi(r)=r^{m} \in L^{\infty}(\mathbb{D})$, where $\alpha>-1, \psi_{k} \in \mathscr{R}_{\alpha}$ and $m \in \mathbb{N}$. If $T_{\varphi} T_{\psi}=T_{\psi} T_{\varphi}$, then $\psi$ is a radial function.

Proof. For every $\lambda \in \mathbb{N}_{0}$, we have

$$
\begin{aligned}
T_{\varphi} z^{\lambda} & =\frac{\Gamma(\lambda+2+\alpha) \Gamma\left(\lambda+\frac{m}{2}+1\right)}{\Gamma\left(\lambda+\frac{m}{2}+2+\alpha\right) \Gamma(\lambda+1)} z^{\lambda} \\
T_{\psi} z^{\lambda} & =\sum_{k=-\infty}^{\infty} \frac{(2 k+2 \lambda+2)^{+} \Gamma(k+\lambda+2+\alpha)}{\Gamma(k+\lambda+2) \Gamma(1+\alpha)} \widehat{\psi}_{\alpha, k}(2 \lambda+k+2) z^{k+\lambda},
\end{aligned}
$$


where $(2 k+2 \lambda+2)^{+}=\max \{2 k+2 \lambda+2,0\}, \widehat{\psi}_{\alpha, k}(z)$ is the Mellin transform of the function $\psi_{k}(r)\left(1-r^{2}\right)^{\alpha}$. Thus

$$
\begin{aligned}
& T_{\psi} T_{\varphi} z^{\lambda} \\
= & \sum_{k=-\infty}^{\infty} \frac{(2 k+2 \lambda+2)^{+} \Gamma(k+\lambda+2+\alpha)}{\Gamma(k+\lambda+2) \Gamma(1+\alpha)} \frac{\Gamma(\lambda+2+\alpha) \Gamma\left(\lambda+\frac{m}{2}+1\right)}{\Gamma\left(\lambda+\frac{m}{2}+2+\alpha\right) \Gamma(\lambda+1)} \\
& \times \widehat{\psi}_{\alpha, k}(2 \lambda+k+2) z^{k+\lambda}, \\
& T_{\varphi} T_{\psi} z^{\lambda} \\
= & \sum_{k=-\infty}^{\infty} \frac{(2 k+2 \lambda+2)^{+} \Gamma(k+\lambda+2+\alpha)}{\Gamma(k+\lambda+2) \Gamma(1+\alpha)} \frac{\Gamma(k+\lambda+2+\alpha) \Gamma\left(k+\lambda+\frac{m}{2}+1\right)}{\Gamma\left(k+\lambda+\frac{m}{2}+2+\alpha\right) \Gamma(k+\lambda+1)} \\
& \times \widehat{\psi}_{\alpha, k}(2 \lambda+k+2) z^{k+\lambda} .
\end{aligned}
$$

Hence for $k+\lambda \geq 0$,

$$
\begin{aligned}
& \frac{(2 k+2 \lambda+2) \Gamma(k+\lambda+2+\alpha)}{\Gamma(k+\lambda+2) \Gamma(1+\alpha)} \frac{\Gamma(\lambda+2+\alpha) \Gamma\left(\lambda+\frac{m}{2}+1\right)}{\Gamma\left(\lambda+\frac{m}{2}+2+\alpha\right) \Gamma(\lambda+1)} \widehat{\psi}_{\alpha, k}(2 \lambda+k+2) \\
= & \frac{(2 k+2 \lambda+2) \Gamma(k+\lambda+2+\alpha)}{\Gamma(k+\lambda+2) \Gamma(1+\alpha)} \frac{\Gamma(k+\lambda+2+\alpha) \Gamma\left(k+\lambda+\frac{m}{2}+1\right)}{\Gamma\left(k+\lambda+\frac{m}{2}+2+\alpha\right) \Gamma(k+\lambda+1)} \\
& \times \widehat{\psi}_{\alpha, k}(2 \lambda+k+2),
\end{aligned}
$$

i.e.,

$$
\begin{aligned}
& \left(\frac{\Gamma(\lambda+2+\alpha) \Gamma\left(\lambda+\frac{m}{2}+1\right)}{\Gamma\left(\lambda+\frac{m}{2}+2+\alpha\right) \Gamma(\lambda+1)}-\frac{\Gamma(k+\lambda+2+\alpha) \Gamma\left(k+\lambda+\frac{m}{2}+1\right)}{\Gamma\left(k+\lambda+\frac{m}{2}+2+\alpha\right) \Gamma(k+\lambda+1)}\right) \\
& \times \widehat{\psi}_{\alpha, k}(2 \lambda+k+2)=0 .
\end{aligned}
$$

For a fixed $k$, we start to discuss the necessary and sufficient conditions that guarantee

$$
\frac{\Gamma(\lambda+2+\alpha) \Gamma\left(\lambda+\frac{m}{2}+1\right)}{\Gamma\left(\lambda+\frac{m}{2}+2+\alpha\right) \Gamma(\lambda+1)}-\frac{\Gamma(k+\lambda+2+\alpha) \Gamma\left(k+\lambda+\frac{m}{2}+1\right)}{\Gamma\left(k+\lambda+\frac{m}{2}+2+\alpha\right) \Gamma(k+\lambda+1)}=0
$$

for all $\lambda \in \mathbb{N}_{0}$. If

$$
\frac{\Gamma(\lambda+2+\alpha) \Gamma\left(\lambda+\frac{m}{2}+1\right)}{\Gamma\left(\lambda+\frac{m}{2}+2+\alpha\right) \Gamma(\lambda+1)}-\frac{\Gamma(k+\lambda+2+\alpha) \Gamma\left(k+\lambda+\frac{m}{2}+1\right)}{\Gamma\left(k+\lambda+\frac{m}{2}+2+\alpha\right) \Gamma(k+\lambda+1)}=0,
$$

since the gamma function is a zero-free analytic function in the right half-plane, then

$$
\frac{\Gamma(\lambda+2+\alpha) \Gamma\left(\lambda+\frac{m}{2}+1\right)}{\Gamma\left(\lambda+\frac{m}{2}+2+\alpha\right) \Gamma(\lambda+1)} \frac{\Gamma\left(k+\lambda+\frac{m}{2}+2+\alpha\right) \Gamma(k+\lambda+1)}{\Gamma(k+\lambda+2+\alpha) \Gamma\left(k+\lambda+\frac{m}{2}+1\right)}=1 .
$$

The left side of the above equation is denoted by $H_{k}(\lambda)$, and it is evident that $H_{k}(\lambda)=1$ when $k=0$. We will prove that $H_{k}(\lambda) \equiv 1$ for all $\lambda \in \mathbb{N}_{0}$ if and only if $k=0$. 
First, we show that if $k>0$, then $H_{k}(\lambda)<1$ for any $\lambda \in \mathbb{N}_{0}$. Let $a_{1}=\lambda+1$, $a_{2}=\lambda+2+\alpha$ and $M=k-1$. Then $a_{1}<a_{2}$ and

$$
H_{k}(\lambda)=\frac{a_{1}\left(a_{1}+1\right) \cdots\left(a_{1}+M\right)\left(a_{2}+\frac{m}{2}\right)\left(a_{2}+\frac{m}{2}+1\right) \cdots\left(a_{2}+\frac{m}{2}+M\right)}{\left(a_{1}+\frac{m}{2}\right)\left(a_{1}+\frac{m}{2}+1\right) \cdots\left(a_{1}+\frac{m}{2}+M\right) a_{2}\left(a_{2}+1\right) \cdots\left(a_{2}+M\right)} .
$$

If $k=1$, then

$$
H_{k}(\lambda)=\frac{a_{1}\left(a_{2}+\frac{m}{2}\right)}{\left(a_{1}+\frac{m}{2}\right) a_{2}}=\frac{a_{1} a_{2}+a_{1} \frac{m}{2}}{a_{1} a_{2}+a_{2} \frac{m}{2}}<1 .
$$

Assume $H_{k}(\lambda)<1$ when $k=M_{0}$, then if $k=M_{0}+1$, we have

$$
\begin{aligned}
& H_{k}(\lambda) \\
= & \frac{a_{1}\left(a_{1}+1\right) \cdots\left(a_{1}+M_{0}\right)\left(a_{2}+\frac{m}{2}\right)\left(a_{2}+\frac{m}{2}+1\right) \cdots\left(a_{2}+\frac{m}{2}+M_{0}\right)}{\left(a_{1}+\frac{m}{2}\right)\left(a_{1}+\frac{m}{2}+1\right) \cdots\left(a_{1}+\frac{m}{2}+M_{0}\right) a_{2}\left(a_{2}+1\right) \cdots\left(a_{2}+M_{0}\right)} \\
= & \frac{a_{1}\left(a_{1}+1\right) \cdots\left(a_{1}+M_{0}-1\right)\left(a_{2}+\frac{m}{2}\right)\left(a_{2}+\frac{m}{2}+1\right) \cdots\left(a_{2}+\frac{m}{2}+M_{0}-1\right)}{\left(a_{1}+\frac{m}{2}\right)\left(a_{1}+\frac{m}{2}+1\right) \cdots\left(a_{1}+\frac{m}{2}+M_{0}-1\right) a_{2}\left(a_{2}+1\right) \cdots\left(a_{2}+M_{0}-1\right)} \\
& \times \frac{\left(a_{1}+M_{0}\right)\left(a_{2}+\frac{m}{2}+M_{0}\right)}{\left(a_{1}+\frac{m}{2}+M_{0}\right)\left(a_{2}+M_{0}\right)} \\
< & \frac{\left(a_{1}+M_{0}\right)\left(a_{2}+\frac{m}{2}+M_{0}\right)}{\left(a_{1}+\frac{m}{2}+M_{0}\right)\left(a_{2}+M_{0}\right)}<1,
\end{aligned}
$$

since $a_{1}<a_{2}$. Then by induction assumption we complete the above proof. Similarly we can obtain that if $k<0$, then $H_{k}(\lambda)>1$ for all $\lambda \in \mathbb{N}_{0}$ with $\lambda+k \geq 0$. Thus $H_{k}(\lambda) \equiv 1$ for all $\lambda \in \mathbb{N}_{0}$ if and only if $k=0$.

Hence we get, if $k=0$, then the equation (1) holds; if $k \neq 0$, then $\widehat{\psi}_{\alpha, k}(2 \lambda+$ $k+2)=0$ for all $\lambda \in \mathbb{N}_{0}$ with $\lambda+k \geq 0$, thus by [18] we get $\psi_{k}(r)=0$. So $\psi\left(r e^{i \theta}\right)=\psi_{0}(r)$.

Theorem 2.2. Let $\psi\left(r e^{i \theta}\right)=\sum_{k=-\infty}^{\infty} e^{i k \theta} \psi_{k}(r)$ and $\varphi(z)=z^{s} z^{-t} \in L^{\infty}(\mathbb{D})$, where $\alpha>-1, \psi_{k} \in \mathscr{R}_{\alpha}, s, t \in \mathbb{N}$ and $s-t \neq 0$. Then $T_{\varphi} T_{\psi}=T_{\psi} T_{\varphi}$ if and only if for every $k$, there exist constants $a_{0}(k), a(k)$ such that

$$
\widehat{\psi}_{\alpha, k}(z)=a_{0}(k) \prod_{p=0}^{s-1} \frac{\Gamma\left(\frac{z}{2 \delta}+a(k)+\frac{p}{\delta}\right)}{\Gamma\left(\frac{z}{2 \delta}+a(k)+\frac{p}{\delta}+\frac{\alpha+1}{\delta}\right)} \prod_{p=\delta}^{s-1} \frac{\Gamma\left(\frac{z}{2 \delta}-a(k)+\frac{p}{\delta}+\frac{\alpha+1}{\delta}\right)}{\Gamma\left(\frac{z}{2 \delta}-a(k)+\frac{p}{\delta}\right)},
$$

where $\delta=s-t, a(k)=\frac{k}{2 \delta}$ and $\widehat{\psi}_{\alpha, k}(z)=\int_{0}^{1} \psi_{k}(r)\left(1-r^{2}\right)^{\alpha} r^{z-1} d r$ is the Mellin transform of the function $\psi_{k}(r)\left(1-r^{2}\right)^{\alpha}$.

Proof. We prefer a polar coordinates representation of our symbols, so let $\psi\left(r e^{i \theta}\right)=\sum_{k=-\infty}^{\infty} e^{i k \theta} \psi_{k}(r)$ and $\varphi\left(r e^{i \theta}\right)=r^{m} e^{i \delta \theta}$, where $m=s+t \geq 0$, $\delta=s-t \in \mathbb{Z}$. Without loss of generality, assume $\delta \geq 0$, for otherwise we could take the adjoints. Case $\delta=0$ leads to Theorem 2.1, so we assume $\delta>0$. If $\lambda \in \mathbb{N}_{0}$, we have

$$
T_{\varphi} z^{\lambda}=\frac{\Gamma(\lambda+\delta+2+\alpha)}{\Gamma(\lambda+\delta+1)} \frac{\Gamma\left(\lambda+\frac{\delta+m}{2}+1\right)}{\Gamma\left(\lambda+\frac{\delta+m}{2}+2+\alpha\right)} z^{\lambda+\delta}
$$


and

$$
T_{\psi} z^{\lambda}=\sum_{k=-\infty}^{\infty} \frac{(2 k+2 \lambda+2)^{+} \Gamma(k+\lambda+2+\alpha)}{\Gamma(k+\lambda+2) \Gamma(1+\alpha)} \widehat{\psi}_{\alpha, k}(2 \lambda+k+2) z^{k+\lambda}
$$

where $(2 \lambda+2 k+2)^{+}=\max \{2 \lambda+2 k+2,0\}, \widehat{\psi}_{\alpha, k}(z)=\int_{0}^{1} \psi_{k}(r)\left(1-r^{2}\right)^{\alpha} r^{z-1} d r$ is the Mellin transform of the function $\psi_{k}(r)\left(1-r^{2}\right)^{\alpha}$.

The equality $T_{\varphi} T_{\psi} z^{\lambda}=T_{\psi} T_{\varphi} z^{\lambda}$ for each $\lambda \in \mathbb{N}_{0}$ together with (2) and (3) gives

$$
\begin{aligned}
& \sum_{k=-\infty}^{\infty} \frac{\Gamma(k+\lambda+\delta+2+\alpha) \Gamma\left(k+\lambda+\frac{m+\delta}{2}+1\right)(2 k+2 \lambda+2)+\Gamma(k+\lambda+2+\alpha)}{\Gamma(k+\lambda+\delta+1) \Gamma\left(k+\lambda+\frac{m+\delta}{2}+2+\alpha\right) \Gamma(k+\lambda+2) \Gamma(1+\alpha)} \\
& \times \widehat{\psi}_{\alpha, k}(2 \lambda+k+2) z^{k+\lambda+\delta} \\
&=\sum_{k=-\infty}^{\infty} \frac{\Gamma(\lambda+\delta+2+\alpha) \Gamma\left(\lambda+\frac{m+\delta}{2}+1\right)(2 k+2 \lambda+2 \delta+2)^{+} \Gamma(k+\lambda+\delta+2+\alpha)}{\Gamma(\lambda+\delta+1) \Gamma\left(\lambda+\frac{m+\delta}{2}+2+\alpha\right) \Gamma(k+\lambda+\delta+2) \Gamma(1+\alpha)} \\
& \quad \times \widehat{\psi}_{\alpha, k}(2 \lambda+2 \delta+k+2) z^{k+\lambda+\delta} .
\end{aligned}
$$

The identity of the two power series implies that for each $\lambda \in \mathbb{N}_{0}$ such that $k+\lambda \geq 0$,

$$
\begin{aligned}
& \widehat{\psi}_{\alpha, k}(2 \lambda+2 \delta+k+2) \\
(4)= & \frac{\Gamma(k+\lambda+2+\alpha) \Gamma(\lambda+\delta+1) \Gamma\left(k+\lambda+\frac{\delta+m}{2}+1\right) \Gamma\left(\lambda+\frac{\delta+m}{2}+2+\alpha\right)}{\Gamma\left(k+\lambda+\frac{\delta+m}{2}+2+\alpha\right) \Gamma\left(\lambda+\frac{\delta+m}{2}+1\right) \Gamma(k+\lambda+1) \Gamma(\lambda+\delta+2+\alpha)} \\
& \times \widehat{\psi}_{\alpha, k}(2 \lambda+k+2)
\end{aligned}
$$

(This also shows that each $T_{e^{i k \theta} \psi_{k}(r)}$ commutes with $T_{\varphi}$ ). Since the integer $\frac{\delta+m}{2}>\max \{1, \delta\}$,

(5)

$$
\widehat{\psi}_{\alpha, k}(2 \lambda+2 \delta+k+2)
$$$$
=\frac{(2 \lambda+2 k+\delta+m) \cdots(2 \lambda+2 k+2)(2 \lambda+\delta+m+2+2 \alpha) \cdots(2 \lambda+2 \delta+4+2 \alpha)}{(2 \lambda+2 k+\delta+m+2+2 \alpha) \cdots(2 \lambda+2 k+4+2 \alpha)(2 \lambda+\delta+m) \cdots(2 \lambda+2 \delta+2)}
$$$$
\times \widehat{\psi}_{\alpha, k}(2 \lambda+k+2) \text {. }
$$

Let $z=2 \lambda+k+2$. Then (5) can be written as

(6)

$$
\begin{aligned}
& \widehat{\psi}_{\alpha, k}(z+2 \delta) \\
= & \frac{(z+k+\delta+m-2) \cdots(z+k)(z-k+\delta+m+2 \alpha) \cdots(z-k+2 \delta+2+2 \alpha)}{(z+k+\delta+m+2 \alpha) \cdots(z+k+2+2 \alpha)(z-k+\delta+m-2) \cdots(z-k+2 \delta)} \\
& \times \widehat{\psi}_{\alpha, k}(z)
\end{aligned}
$$


for positive integers $z \geq|k|+2$. If $M>0$ denotes a bound for $\psi$, then for a fixed radius $r_{0}<1$,

$$
\int_{0}^{2 \pi} \psi\left(r_{0} e^{i \theta}\right) e^{-i k \theta} \frac{d \theta}{2 \pi}=\psi_{k}\left(r_{0}\right) .
$$

Thus $\left|\psi_{k}\left(r_{0}\right)\right| \leq M$, which implies that each $\psi_{k}$ is also bounded on $[0,1)$, since $r_{0}$ is arbitrary. In [18] we also note that for each $k$, the function $\widehat{\psi}_{\alpha, k}(z)$ is analytic in the right half-plane $\{z \in \mathbb{C}: \operatorname{Re} z>2\}$. Fix $k$, then

$$
\begin{aligned}
\left|\widehat{\psi}_{\alpha, k}(z)\right| & \leq \int_{0}^{1}\left|\psi_{k}(r)\right|\left|r^{z-1}\right|\left(1-r^{2}\right)^{\alpha} d r \\
& \leq M \int_{0}^{1} r^{x-1}\left(1-r^{2}\right)^{\alpha} d r \\
& \leq M \int_{0}^{1} r\left(1-r^{2}\right)^{\alpha} d r=\frac{M}{2(\alpha+1)}
\end{aligned}
$$

and $\left|\widehat{\psi}_{\alpha, k}(z+2 \delta)\right| \leq \frac{M}{2(\alpha+1)}$ if $x \geq 2$. Therefore in some right half-plane, say Re $z \geq N, N>0$, the function

$$
\widehat{\psi}_{\alpha, k}(z+2 \delta)-\frac{(z+k+\delta+m-2) \cdots(z+k)(z-k+\delta+m+2 \alpha) \cdots(z-k+2 \delta+2+2 \alpha)}{(z+k+\delta+m+2 \alpha) \cdots(z+k+2+2 \alpha)(z-k+\delta+m-2) \cdots(z-k+2 \delta)} \widehat{\psi}_{\alpha, k}(z)
$$

is bounded and analytic. The equation (6) shows that this function has zeros at the integers lying in the right half-plane. By [18] we know that a bounded analytic function in the half-plane cannot have zeros $\left\{\alpha_{j}\right\}$ that form an arithmetic sequence, because the Blaschke condition

$$
\prod_{j}\left|\frac{1-\alpha_{j}}{1+\alpha_{j}}\right|>0
$$

is not satisfied. Thus for $\operatorname{Re} z>2$

$$
\begin{aligned}
& \widehat{\psi}_{\alpha, k}(z+2 \delta) \\
= & \frac{(z+k+\delta+m-2) \cdots(z+k)(z-k+\delta+m+2 \alpha) \cdots(z-k+2 \delta+2+2 \alpha)}{(z+k+\delta+m+2 \alpha) \cdots(z+k+2+2 \alpha)(z-k+\delta+m-2) \cdots(z-k+2 \delta)} \\
& \times \widehat{\psi}_{\alpha, k}(z) .
\end{aligned}
$$

Observe that

$$
\begin{aligned}
& \frac{(z+k+\delta+m-2) \cdots(z+k)(z-k+\delta+m+2 \alpha) \cdots(z-k+2 \delta+2+2 \alpha)}{(z+k+\delta+m+2 \alpha) \cdots(z+k+2+2 \alpha)(z-k+\delta+m-2) \cdots(z-k+2 \delta)} \\
= & \prod_{p=0}^{\frac{\delta+m}{2}-1} \frac{z+k+2 p}{z+k+2 p+2+2 \alpha} \prod_{p=\delta}^{\frac{\delta+m}{2}-1} \frac{z-k+2 p+2+2 \alpha}{z-k+2 p} \\
= & \prod_{p=0}^{\frac{\delta+m}{2}-1} \frac{\frac{z}{2 \delta}+\frac{k}{2 \delta}+\frac{p}{\delta}}{\frac{z}{2 \delta}+\frac{k}{2 \delta}+\frac{p}{\delta}+\frac{1+\alpha}{\delta}} \prod_{p=\delta}^{\frac{\delta+m}{2}-1} \frac{\frac{z}{2 \delta}-\frac{k}{2 \delta}+\frac{p}{\delta}+\frac{1+\alpha}{\delta}}{\frac{z}{2 \delta}-\frac{k}{2 \delta}+\frac{p}{\delta}} .
\end{aligned}
$$


So, for $\operatorname{Re} z>2$,

$$
\begin{aligned}
& \widehat{\psi}_{\alpha, k}(z+2 \delta) \\
= & \widehat{\psi}_{\alpha, k}(z) \prod_{p=0}^{\frac{\delta+m}{2}-1} \frac{\frac{z}{2 \delta}+a(k)+\frac{p}{\delta}}{\frac{z}{2 \delta}+a(k)+\frac{p}{\delta}+\frac{1+\alpha}{\delta}} \prod_{p=\delta}^{\frac{\delta+m}{2}-1} \frac{\frac{z}{2 \delta}-a(k)+\frac{p}{\delta}+\frac{1+\alpha}{\delta}}{\frac{z}{2 \delta}-a(k)+\frac{p}{\delta}},
\end{aligned}
$$

where $a(k)=k / 2 \delta$.

Let $\Gamma(z)$ denote the gamma function and let

$$
F(z)=\prod_{p=0}^{\frac{\delta+m}{2}-1} \frac{\Gamma\left(\frac{z}{2 \delta}+a(k)+\frac{p}{\delta}\right)}{\Gamma\left(\frac{z}{2 \delta}+a(k)+\frac{p}{\delta}+\frac{1+\alpha}{\delta}\right)} \prod_{p=\delta}^{\frac{\delta+m}{2}-1} \frac{\Gamma\left(\frac{z}{2 \delta}-a(k)+\frac{p}{\delta}+\frac{1+\alpha}{\delta}\right)}{\Gamma\left(\frac{z}{2 \delta}-a(k)+\frac{p}{\delta}\right)} .
$$

Using the well-known identity $\Gamma(z+1)=z \Gamma(z)$, we can easily obtain that

$$
F(z+2 \delta)
$$

$$
=F(z) \prod_{p=0}^{\frac{\delta+m}{2}-1} \frac{\frac{z}{2 \delta}+a(k)+\frac{p}{\delta}}{\frac{z}{2 \delta}+a(k)+\frac{p}{\delta}+\frac{1+\alpha}{\delta}} \prod_{p=\delta}^{\frac{\delta+m}{2}-1} \frac{\frac{z}{2 \delta}-a(k)+\frac{p}{\delta}+\frac{1+\alpha}{\delta}}{\frac{z}{2 \delta}-a(k)+\frac{p}{\delta}} .
$$

From (7) and (8) we can get

$$
\frac{\widehat{\psi}_{\alpha, k}(z+2 \delta)}{F(z+2 \delta)}=\frac{\widehat{\psi}_{\alpha, k}(z)}{F(z)} \quad \text { for } \quad \operatorname{Re} z>2 .
$$

Hence the function $\widehat{\psi}_{\alpha, k}(z) / F(z)$ is a periodic function with a period $2 \delta$ if $\operatorname{Re} z>2$. From the asymptotic expression of the logarithm of the gamma function (see, for example, [24, p. 251]),

$$
\log \Gamma(z)=\left(z-\frac{1}{2}\right) \log (z)-z+\frac{1}{2} \log 2 \pi+O\left(\frac{1}{z}\right),
$$

we see that

$$
\Gamma(z)=\left[e^{(z-1 / 2) \log (z)-z+1 / 2 \log 2 \pi}\right]\left(1+O\left(\frac{1}{z}\right)\right)
$$

for Re $z>0$. Hence

$$
\prod_{p=0}^{\frac{\delta+m}{2}-1} \frac{\Gamma\left(z+a+\frac{p}{\delta}\right)}{\Gamma\left(z+a+\frac{p}{\delta}+\frac{1+\alpha}{\delta}\right)} \prod_{p=\delta}^{\frac{\delta+m}{2}-1} \frac{\Gamma\left(z-a+\frac{p}{\delta}+\frac{1+\alpha}{\delta}\right)}{\Gamma\left(z-a+\frac{p}{\delta}\right)}
$$




$$
\begin{aligned}
= & \prod_{p=\delta}^{\frac{\delta+m}{2}-1} \frac{\Gamma\left(z+a+\frac{p}{\delta}\right) \Gamma\left(z-a+\frac{p}{\delta}+\frac{1+\alpha}{\delta}\right)}{\Gamma\left(z+a+\frac{p}{\delta}+\frac{1+\alpha}{\delta}\right) \Gamma\left(z-a+\frac{p}{\delta}\right)} \prod_{p=0}^{\delta-1} \frac{\Gamma\left(z+a+\frac{p}{\delta}\right)}{\Gamma\left(z+a+\frac{p}{\delta}+\frac{1+\alpha}{\delta}\right)} \\
= & \prod_{p=0}^{\frac{\delta+m}{2}-1} \exp \left\{\left(z+a+\frac{p}{\delta}-\frac{1}{2}\right) \log \left(z+a+\frac{p}{\delta}\right)-\left(z+a+\frac{p}{\delta}\right)+\frac{1}{2} \log 2 \pi\right. \\
& +\left(z-a+\frac{p}{\delta}+\frac{\alpha+1}{\delta}-\frac{1}{2}\right) \log \left(z-a+\frac{p}{\delta}+\frac{\alpha+1}{\delta}\right)-\left(z-a+\frac{p}{\delta}+\frac{\alpha+1}{\delta}\right)+\frac{1}{2} \log 2 \pi \\
& -\left(z+a+\frac{p}{\delta}+\frac{\alpha+1}{\delta}-\frac{1}{2}\right) \log \left(z+a+\frac{p}{\delta}+\frac{\alpha+1}{\delta}\right)+\left(z+a+\frac{p}{\delta}+\frac{\alpha+1}{\delta}\right)-\frac{1}{2} \log 2 \pi \\
& \left.-\left(z-a+\frac{p}{\delta}-\frac{1}{2}\right) \log \left(z-a+\frac{p}{\delta}\right)+\left(z-a+\frac{p}{\delta}\right)-\frac{1}{2} \log 2 \pi\right\} \\
& \times \prod_{p=0}^{\delta-1} \exp \left\{\left(z+a+\frac{p}{\delta}-\frac{1}{2}\right) \log \left(z+a+\frac{p}{\delta}\right)-\left(z+a+\frac{p}{\delta}\right)+\frac{1}{2} \log 2 \pi\right. \\
& \left.-\left(z+a+\frac{p}{\delta}+\frac{\alpha+1}{\delta}-\frac{1}{2}\right) \log \left(z+a+\frac{p}{\delta}+\frac{\alpha+1}{\delta}\right)+\left(z+a+\frac{p}{\delta}+\frac{\alpha+1}{\delta}\right)-\frac{1}{2} \log 2 \pi\right\} \\
& \times\left\{1+O\left(\frac{1}{z}\right)\right\} .
\end{aligned}
$$

Note that $\log \left(z+a+\frac{p}{\delta}\right)=\log z+\log \left(1+\left(a+\frac{p}{\delta}\right) / z\right)=\log z+O\left(\frac{1}{z}\right)$, so the expression above becomes

$$
\begin{aligned}
& \prod_{p=0}^{\frac{\delta+m}{2}-1} \frac{\Gamma\left(z+a+\frac{p}{\delta}\right)}{\Gamma\left(z+a+\frac{p}{\delta}+\frac{1+\alpha}{\delta}\right)} \prod_{p=\delta}^{\frac{\delta+m}{2}-1} \frac{\Gamma\left(z-a+\frac{p}{\delta}+\frac{1+\alpha}{\delta}\right)}{\Gamma\left(z-a+\frac{p}{\delta}\right)} \\
= & \prod_{p=0}^{\delta-1} e^{-\frac{\alpha+1}{\delta} \log z+\frac{\alpha+1}{\delta}} \cdot\left\{1+O\left(\frac{1}{z}\right)\right\}=\frac{e^{\alpha+1}}{z^{\alpha+1}} \cdot\left(1+O\left(\frac{1}{z}\right)\right) .
\end{aligned}
$$

Thus $F(z)=\frac{(2 \delta)^{\alpha+1} e^{\alpha+1}}{z^{\alpha+1}} \cdot\left(1+O\left(\frac{1}{z}\right)\right)$ for Re $z>0$ large, and that implies that

$$
\frac{\widehat{\psi}_{\alpha, k}(z)}{F(z)}=O\left(|z|^{\alpha+1}\right) \quad \text { if } \quad \operatorname{Re} z \geq N \text { large. }
$$

It is well-known that the gamma function is a zero-free function in the right half-plane. Thus $F(z)$ is analytic for $\operatorname{Re} z$ large and hence $\widehat{\psi}_{\alpha, k}(z) / F(z)$ is analytic for $\operatorname{Re} z \geq N$. Because it is periodic, we can extend it to the whole plane, so $\widehat{\psi}_{\alpha, k}(z) / F(z)$ is an entire function of period $2 \delta$. For every $z \in \mathbb{C}$, $z=x+i y$, there exists a $k_{z} \in \mathbb{Z}$ such that

$$
x+2\left(k_{z}-1\right) \delta \leq N \leq x+2 k_{z} \delta
$$

then

$$
\left|2 k_{z} \delta\right| \leq \max \{|N-x|,|N-x+2 \delta|\} \leq N+|x|+2 \delta \leq N+|z|+2 \delta .
$$


For that $z$, we have

$$
\begin{aligned}
\left|\frac{\widehat{\psi}_{\alpha, k}(z)}{F(z)}\right| & =\left|\frac{\widehat{\psi}_{\alpha, k}\left(z+2 k_{z} \delta\right)}{F\left(z+2 k_{z} \delta\right)}\right| \leq C\left|z+2 k_{z} \delta\right|^{\alpha+1} \leq C\left(|z|+\left|2 k_{z} \delta\right|\right)^{\alpha+1} \\
& \leq C(2|z|+N+2 \delta)^{\alpha+1} \leq C(2|z|+N+2 \delta)^{[\alpha]+2}
\end{aligned}
$$

where $[\alpha]$ is the integral part of $\alpha$. By the fact that if $n$ is a positive integer and $x, y>0$, then $\left(\frac{x+y}{2}\right)^{n} \leq \frac{x^{n}+y^{n}}{2}$, we have, for $|z| \geq N+2 \delta$

$$
\left|\frac{\widehat{\psi}_{\alpha, k}(z)}{F(z)}\right|=\left|\frac{\widehat{\psi}_{\alpha, k}\left(z+2 k_{z} \delta\right)}{F\left(z+2 k_{z} \delta\right)}\right| \leq C\left|z+2 k_{z} \delta\right|^{\alpha+1} \leq C_{0}|z|^{[\alpha]+2}
$$

for some constant $C_{0}$. So by $[5] \frac{\widehat{\psi}_{\alpha, k}(z)}{F(z)}$ is a polynomial of degree $\leq[\alpha]+2$.

Claim 2.3. If $f$ is a polynomial and a periodic function with period $t_{0}>0$, then $f$ is a constant.

Proof. Suppose $f(z)=\sum_{p=0}^{n} a_{p} z^{p}$. If $f$ is not a constant, then there is a complex number $q$ such that $f(q)=0$. Because $f$ is periodic with period $t_{0}$, we have $f\left(q+p t_{0}\right)=0$ for any $p \in \mathbb{Z}$, i.e., $\left\{q+p t_{0}: p \in \mathbb{Z}\right\}$ are zeros of $f$. But the degree of $f$ is $n$, by the Fundamental Theorem of Algebra we know that $f$ just has $n$ zeros, which leads to a contradiction.

Since $\frac{\widehat{\psi}_{\alpha, k}(z)}{F(z)}$ is periodic, by Claim 2.3 it must be constant. Therefore

$$
\frac{\widehat{\psi}_{\alpha, k}(z)}{F(z)}=a_{0}(k)
$$

i.e.,

$\widehat{\psi}_{\alpha, k}(z)=a_{0}(k) \prod_{p=0}^{\frac{\delta+m}{2}-1} \frac{\Gamma\left(\frac{z}{2 \delta}+a(k)+\frac{p}{\delta}\right)}{\Gamma\left(\frac{z}{2 \delta}+a(k)+\frac{p}{\delta}+\frac{\alpha+1}{\delta}\right)} \prod_{p=\delta}^{\frac{\delta+m}{2}-1} \frac{\Gamma\left(\frac{z}{2 \delta}-a(k)+\frac{p}{\delta}+\frac{\alpha+1}{\delta}\right)}{\Gamma\left(\frac{z}{2 \delta}-a(k)+\frac{p}{\delta}\right)}$.

Since $m=s+t, \delta=s-t$, then

$$
\widehat{\psi}_{\alpha, k}(z)=a_{0}(k) \prod_{p=0}^{s-1} \frac{\Gamma\left(\frac{z}{2 \delta}+a(k)+\frac{p}{\delta}\right)}{\Gamma\left(\frac{z}{2 \delta}+a(k)+\frac{p}{\delta}+\frac{\alpha+1}{\delta}\right)} \prod_{p=\delta}^{s-1} \frac{\Gamma\left(\frac{z}{2 \delta}-a(k)+\frac{p}{\delta}+\frac{\alpha+1}{\delta}\right)}{\Gamma\left(\frac{z}{2 \delta}-a(k)+\frac{p}{\delta}\right)} .
$$

Hence the necessary is completed. Conversely, if the conditions of Theorem 2.2 are satisfied, then equation (4) holds, and therefor $T_{\varphi}$ commutes with $T_{\psi}$.

From the expression of $\widehat{\psi}_{\alpha, k}(z)$, we can see, if $\alpha$ is a decimal, then $\widehat{\psi}_{\alpha, k}(z)$ is not a rational function. The reason is that $\left(\left(a(k)+\frac{p_{1}}{\delta}\right)-\left(a(k)+\frac{p_{2}}{\delta}+\frac{\alpha+1}{\delta}\right)\right)$ is not an integer and the terms of $\Gamma\left(\frac{z}{2 \delta}+a(k)+\frac{p_{1}}{\delta}\right)$ is not equivalent to that of $\Gamma\left(\frac{z}{2 \delta}-a(k)+\frac{p_{3}}{\delta}\right)$, where $p_{1}=0,1, \ldots, s-1 ; p_{2}=0,1, \ldots, s-1 ; p_{3}=\delta, \ldots, s-1$. 
If $\alpha$ is an integer, similarly we can get an another expression of $\widehat{\psi}_{\alpha, k}(z)$ as follows:

$$
\widehat{\psi}_{\alpha, k}(z)=a_{0}^{\prime}(k) \prod_{p=0}^{\alpha} \frac{\Gamma\left(\frac{z}{2 \delta}+a(k)+\frac{p}{\delta}\right) \Gamma\left(\frac{z}{2 \delta}+b(k)+\frac{p}{\delta}\right)}{\Gamma\left(\frac{z}{2 \delta}+c(k)+\frac{p}{\delta}\right) \Gamma\left(\frac{z}{2 \delta}+d(k)+\frac{p}{\delta}\right)},
$$

where $a(k)=k / 2 \delta, b(k)=(m+\delta-k) / 2 \delta, c(k)=(2 \delta-k) / 2 \delta, d(k)=(m+$ $\delta+k) / 2 \delta$, and

$$
a(k)+b(k)-c(k)-d(k)=-1 .
$$

Theorem 2.4. Let $\psi_{k}(r)$ be a bounded function on $[0,1)$ for each $k \in \mathbb{Z}$, that satisfies

$$
\widehat{\psi}_{\alpha, k}(z)=\prod_{p=0}^{\alpha} \frac{\Gamma\left(\frac{z}{2 \delta}+a(k)+\frac{p}{\delta}\right) \Gamma\left(\frac{z}{2 \delta}+b(k)+\frac{p}{\delta}\right)}{\Gamma\left(\frac{z}{2 \delta}+c(k)+\frac{p}{\delta}\right) \Gamma\left(\frac{z}{2 \delta}+d(k)+\frac{p}{\delta}\right)},
$$

where $a(k), b(k), c(k)$ and $d(k)$ are as in equality (12) satisfying the equation (13); $\delta \in \mathbb{N}$. Then $\widehat{\psi}_{\alpha, k}(z)$ is a rational function if and only if one of the following conditions is satisfied:

(1) $\alpha+1=M \delta$, where $M \in \mathbb{N}$;

(2) For any $M \in \mathbb{N}, \alpha+1 \neq M \delta$, but one of $a(k)-c(k), a(k)-d(k)$, $b(k)-c(k)$ and $b(k)-d(k)$ is an integer.

In this case, $\widehat{\psi}_{\alpha, k}(z)$ is a proper rational function with the degree of the denominator by $(\alpha+1)$ bigger than the degree of the numerator. Moreover,

(1) if $a(k)=c(k)$, then $\psi_{\delta}(r)$ has the form $\psi_{\delta}(r)=\frac{2 \delta^{\alpha+1}}{(\alpha+1) !} r^{m}$

(2) if $a(k)=c(k)-1$, then $\psi_{0}(r)$ has the form $\psi_{0}(r)=\frac{2 \delta^{\alpha+1}}{(\alpha+1) !}$.

Proof. Let $a=a(k), b=b(k), c=c(k)$ and $d=d(k)$ for simplicity. Suppose $\widehat{\psi}_{\alpha, k}(z)$ is a rational function, then it has at most finitely many poles. Since the gamma function has no zeros, the poles of $\widehat{\psi}_{\alpha, k}(z)$ come from the poles of $\Gamma\left(\frac{z}{2 \delta}+a+\frac{p}{\delta}\right)$ and $\Gamma\left(\frac{z}{2 \delta}+b+\frac{p}{\delta}\right)$, where $p=0,1, \ldots, \alpha$. But the gamma function has poles at $-1,-2, \ldots$, then all but finitely many of them must be cancelled by the poles of $\Gamma\left(\frac{z}{2 \delta}+c+\frac{p}{\delta}\right)$ or $\Gamma\left(\frac{z}{2 \delta}+d+\frac{p}{\delta}\right)$, where $p=0,1, \ldots, \alpha$. Observe that

$$
a-c=\frac{k}{2 \delta}-\frac{2 \delta-k}{2 \delta}=-1+\frac{k}{\delta}, \quad d-b=\frac{k+\delta+m}{2 \delta}-\frac{\delta+m-k}{2 \delta}=\frac{k}{\delta},
$$

then $a-c$ and $b-d$ are integers if and only if $k / \delta$ is an integer. Let $\xi=\frac{z}{2 \delta}$, then (14) can be written as

$$
\widehat{\psi}_{\alpha, k}(z)=\prod_{p=0}^{\alpha} \frac{\Gamma\left(\xi+c-1+\frac{k}{\delta}+\frac{p}{\delta}\right) \Gamma\left(\xi+b+\frac{p}{\delta}\right)}{\Gamma\left(\xi+c+\frac{p}{\delta}\right) \Gamma\left(\xi+b+\frac{k}{\delta}+\frac{p}{\delta}\right)} .
$$

If $\alpha+1=M \delta$, where $M \in \mathbb{N}$, because for each $p \in\{0,1, \ldots, \alpha\}$ there exists a $q \in\{0,1, \ldots, \alpha\}$ such that $\frac{q-k-p}{\delta} \in \mathbb{Z}$, so $\widehat{\psi}_{\alpha, k}(z)$ is a rational function. 
If $\alpha+1=M \delta+r$, where $M \in \mathbb{N}, r=1,2, \ldots, \delta-1$, by the above discussion the equation (15) can be written as

$$
\widehat{\psi}_{\alpha, k}(z)=f(z) \prod_{p=M \delta}^{M \delta+r-1} \frac{\Gamma\left(\xi+c+\frac{k}{\delta}+\frac{p}{\delta}\right) \Gamma\left(\xi+b+\frac{p}{\delta}\right)}{\Gamma\left(\xi+c+\frac{p}{\delta}\right) \Gamma\left(\xi+b+\frac{k}{\delta}+\frac{p}{\delta}\right)},
$$

where $f(z)$ is a rational function. So $\widehat{\psi}_{\alpha, k}(z)$ is a rational function if and only if $\prod_{p=M \delta}^{M \delta+r-1} \frac{\Gamma\left(\xi+c+\frac{k}{\delta}+\frac{p}{\delta}\right) \Gamma\left(\xi+b+\frac{p}{\delta}\right)}{\Gamma\left(\xi+c+\frac{p}{\delta}\right) \Gamma\left(\xi+b+\frac{k}{\delta}+\frac{p}{\delta}\right)}$ is a rational function if and only if $\prod_{p=0}^{r-1} \frac{\Gamma\left(\xi+c+\frac{k}{\delta}+\frac{p}{\delta}\right) \Gamma\left(\xi+b+\frac{p}{\delta}\right)}{\Gamma\left(\xi+c+\frac{p}{\delta}\right) \Gamma\left(\xi+b+\frac{k}{\delta}+\frac{p}{\delta}\right)}$ is a rational function.

Now let us discuss the necessary and sufficient conditions that the function $\prod_{p=0}^{r_{1}} \frac{\Gamma\left(\xi+c+\frac{k}{\delta}+\frac{p}{\delta}\right) \Gamma\left(\xi+b+\frac{p}{\delta}\right)}{\Gamma\left(\xi+c+\frac{p}{\delta}\right) \Gamma\left(\xi+b+\frac{k}{\delta}+\frac{p}{\delta}\right)}$ is a rational function, where $r_{1}=0,1, \ldots, \delta-2$.

Define a function $g: \mathbb{N} \longrightarrow \mathbb{N}$ as follows:

$$
g(n)=r_{0}, \quad \text { if }\left(n-r_{0}, \delta\right)=\delta, \quad r_{0}=0,1, \ldots, \delta-1,
$$

where $(a, b)$ denotes the greatest common divisor of $a$ and $b$.

Let $M_{0}=\left\{0,1, \ldots, r_{1}\right\}$ and

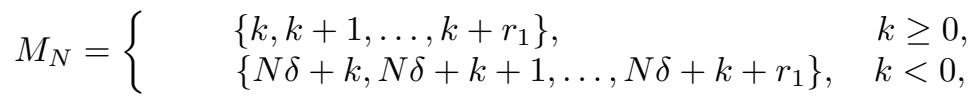

where $N \in \mathbb{N}$ with $N \delta+k \geq 0$. Before continuing the proof we need the following two claims.

Claim 2.5. Suppose $g_{1}(\xi)=\prod_{p=0}^{r_{1}} \frac{\Gamma\left(\xi+b+\frac{p}{\delta}\right)}{\Gamma\left(\xi+c+\frac{p}{\delta}\right)}$, where $r_{1}=0,1, \ldots, \delta-2$. Then $g_{1}(\xi)$ is a rational function if and only if $b-c$ is an integer.

Proof. Suppose $g_{1}(\xi)$ is a rational function. Then for each $p_{1} \in\left\{0,1, \ldots, r_{1}\right\}$ there is a $p_{2} \in\left\{0,1, \ldots, r_{1}\right\}$ such that $\left(c+\frac{p_{2}}{\delta}\right)-\left(b+\frac{p_{1}}{\delta}\right) \in \mathbb{Z}$, so there exist $k_{0}, k_{1} \in\left\{0,1, \ldots, r_{1}\right\}$ and $N_{0}, N_{1} \in \mathbb{Z}$ such that

$$
\begin{aligned}
& b=c+\frac{k_{0}}{\delta}+N_{0}, \\
& c=b+\frac{k_{1}}{\delta}+N_{1} .
\end{aligned}
$$

By (17) and $\left(17^{\prime}\right)$ we get

$$
b=b+\frac{k_{0}+k_{1}}{\delta}+N_{0}+N_{1}
$$

so $\frac{k_{0}+k_{1}}{\delta} \in \mathbb{Z}$, then $k_{0}+k_{1}=0$ or $k_{0}+k_{1}=\delta$, since $0 \leq k_{0}+k_{1} \leq 2 \delta-2$.

If $k_{0}+k_{1}=0$, then we can obtain that $b-c$ is an integer.

If $k_{0}+k_{1}=\delta$, by (17) we get

$$
\begin{aligned}
b & =c+\frac{k_{0}}{\delta}+N_{0}, \\
b+\frac{1}{\delta} & =c+\frac{k_{0}+1}{\delta}+N_{0},
\end{aligned}
$$




$$
b+\frac{r_{1}-k_{0}}{\delta}=c+\frac{r_{1}}{\delta}+N_{0} .
$$

By $\left(17^{\prime}\right)$ we have

$$
\begin{aligned}
c & =b+\frac{k_{1}}{\delta}+N_{1}, \\
c+\frac{1}{\delta} & =b+\frac{k_{1}+1}{\delta}+N_{1}, \\
& \vdots \\
c+\frac{r_{1}-k_{1}}{\delta} & =b+\frac{r_{1}}{\delta}+N_{1} .
\end{aligned}
$$

Since $0 \leq r_{1} \leq \delta-2$, then there exists a $p_{0}$ with $0<r_{1}-k_{1}+1 \leq p_{0} \leq k_{0}-1 \leq r_{1}$ such that $b+\frac{r_{1}-k_{0}+1}{\delta}=c+\frac{p_{0}}{\delta}+N_{2}$, where $N_{2} \in \mathbb{Z}$. Since $b+\frac{r_{1}-k_{0}+1}{\delta}=$ $c+\frac{r_{1}+1}{\delta}+N_{0}$, then $c+\frac{p_{0}}{\delta}+N_{2}=c+\frac{r_{1}+1}{\delta}+N_{0}$, i.e., $\frac{r_{1}+1-p_{0}}{\delta} \in \mathbb{Z}$. However $0<r_{1}-k_{1}+1 \leq p_{0} \leq k_{0}-1 \leq r_{1} \leq \delta-2$, which leads to a contradiction.

It is evident that the converse is also true.

Claim 2.6. Suppose $g_{2}(\xi)=\prod_{p=r_{2}}^{\delta-1} \frac{\Gamma\left(\xi+b+\frac{p}{\delta}\right)}{\Gamma\left(\xi+c+\frac{p}{\delta}\right)}$, where $r_{2}=0,1, \ldots, \delta-1$. Then $g_{2}(\xi)$ is a rational function if and only if $b-c$ is an integer.

Proof. The proof is similar to Claim 2.5.

Now continuing the proof of Theorem 2.4. Without loss of generality, let $0 \leq k \leq \delta-1$.

If $k=0$, then $\prod_{p=0}^{r_{1}} \frac{\Gamma\left(\xi+c+\frac{k}{\delta}+\frac{p}{\delta}\right) \Gamma\left(\xi+b+\frac{p}{\delta}\right)}{\Gamma\left(\xi+c+\frac{p}{\delta}\right) \Gamma\left(\xi+b+\frac{k}{\delta}+\frac{p}{\delta}\right)}$ is a rational function.

If $k \neq 0$ and $M_{0} \bigcap g\left(M_{N}\right)=\emptyset$, then $\prod_{p=0}^{r_{1}} \frac{\Gamma\left(\xi+c+\frac{k}{\delta}+\frac{p}{\delta}\right) \Gamma\left(\xi+b+\frac{p}{\delta}\right)}{\Gamma\left(\xi+c+\frac{p}{\delta}\right) \Gamma\left(\xi+b+\frac{k}{\delta}+\frac{p}{\delta}\right)}$ is a rational function if and only if $\prod_{p=0}^{r_{1}} \frac{\Gamma\left(\xi+b+\frac{p}{\delta}\right)}{\Gamma\left(\xi+c+\frac{p}{\delta}\right)}$ and $\prod_{p=0}^{r_{1}} \frac{\Gamma\left(\xi+c+\frac{p+k}{\delta}\right)}{\Gamma\left(\xi+b+\frac{p+k}{\delta}\right)}$ are both rational functions (otherwise, $\prod_{p=0}^{r_{1}} \frac{\Gamma\left(\xi+c+\frac{k}{\delta}+\frac{p}{\delta}\right) \Gamma\left(\xi+b+\frac{p}{\delta}\right)}{\Gamma\left(\xi+c+\frac{p}{\delta}\right) \Gamma\left(\xi+b+\frac{k}{\delta}+\frac{p}{\delta}\right)}$ has infinitely many poles, but a rational function only has finitely many poles). By Claim 2.5 we have that $\prod_{p=0}^{r_{1}} \frac{\Gamma\left(\xi+b+\frac{p}{\delta}\right)}{\Gamma\left(\xi+c+\frac{p}{\delta}\right)}$ is a rational function if and only if $b-c$ is an integer, and when $b-c$ is an integer, $\prod_{p=0}^{r_{1}} \frac{\Gamma\left(\xi+c+\frac{p+k}{\delta}\right)}{\Gamma\left(\xi+b+\frac{p+k}{\delta}\right)}$ is a rational function. So we obtain that $\prod_{p=0}^{r_{1}} \frac{\Gamma\left(\xi+c+\frac{k}{\delta}+\frac{p}{\delta}\right) \Gamma\left(\xi+b+\frac{p}{\delta}\right)}{\Gamma\left(\xi+c+\frac{p}{\delta}\right) \Gamma\left(\xi+b+\frac{k}{\delta}+\frac{p}{\delta}\right)}$ is a rational function if and only if $b-c$ is an integer.

If $k \neq 0$ and $M_{0} \cap g\left(M_{N}\right) \neq \emptyset$, let

$$
\begin{aligned}
& q_{1}=\left\{\begin{array}{cc}
0, & \text { if } r_{1}+k \leq \delta-1, \\
g\left(r_{1}+k\right)+1, & \text { if } r_{1}+k \geq \delta,
\end{array}\right. \\
& q_{2}=\left\{\begin{array}{cc}
k-1, & \text { if } k \leq r_{1}, \\
r_{1}, & \text { if } k>r_{1},
\end{array}\right.
\end{aligned}
$$




$$
\begin{aligned}
q_{3} & =\left\{\begin{array}{cl}
r_{1}+1, & \text { if } k \leq r_{1}, \\
k, & \text { if } k>r_{1},
\end{array}\right. \\
q_{4} & =\left\{\begin{array}{cl}
k+r_{1}, & \text { if } k+r_{1} \leq \delta-1, \\
\delta-1, & \text { if } k+r_{1} \geq \delta,
\end{array}\right.
\end{aligned}
$$

then

$$
\begin{aligned}
& \prod_{p=0}^{r_{1}} \frac{\Gamma\left(\xi+c+\frac{k}{\delta}+\frac{p}{\delta}\right) \Gamma\left(\xi+b+\frac{p}{\delta}\right)}{\Gamma\left(\xi+c+\frac{p}{\delta}\right) \Gamma\left(\xi+b+\frac{k}{\delta}+\frac{p}{\delta}\right)} \\
= & f_{1}(\xi) \frac{\prod_{p=q_{3}}^{q_{4}} \Gamma\left(\xi+c+\frac{p}{\delta}\right) \prod_{p=q_{1}}^{q_{2}} \Gamma\left(\xi+b+\frac{p}{\delta}\right)}{\prod_{p=q_{3}}^{q_{4}} \Gamma\left(\xi+b+\frac{p}{\delta}\right) \prod_{p=q_{1}}^{q_{2}} \Gamma\left(\xi+c+\frac{p}{\delta}\right)} \\
= & f_{1}(\xi) \prod_{p=q_{3}}^{q_{4}} \frac{\Gamma\left(\xi+c+\frac{p}{\delta}\right)}{\Gamma\left(\xi+b+\frac{p}{\delta}\right)} \prod_{p=q_{1}}^{q_{2}} \frac{\Gamma\left(\xi+b+\frac{p}{\delta}\right)}{\Gamma\left(\xi+c+\frac{p}{\delta}\right)},
\end{aligned}
$$

where $f_{1}(\xi)$ is a rational function. Since $q_{1} \leq q_{2}<q_{3} \leq q_{4} \leq \delta-1$, then $\prod_{p=0}^{r_{1}} \frac{\Gamma\left(\xi+c+\frac{k}{\delta}+\frac{p}{\delta}\right) \Gamma\left(\xi+b+\frac{p}{\delta}\right)}{\Gamma\left(\xi+c+\frac{p}{\delta}\right) \Gamma\left(\xi+b+\frac{k}{\delta}+\frac{p}{\delta}\right)}$ is a rational function if and only if $\prod_{p=q_{3}}^{q_{4}} \frac{\Gamma\left(\xi+c+\frac{p}{\delta}\right)}{\Gamma\left(\xi+b+\frac{p}{\delta}\right)}$ and $\prod_{p=q_{1}}^{q_{2}} \frac{\Gamma\left(\xi+b+\frac{p}{\delta}\right)}{\Gamma\left(\xi+c+\frac{p}{\delta}\right)}$ are rational functions. By Claims 2.5 and 2.6 we have that $\prod_{p=q_{3}}^{q_{4}} \frac{\Gamma\left(\xi+c+\frac{p}{\delta}\right)}{\Gamma\left(\xi+b+\frac{p}{\delta}\right)}$ and $\prod_{p=q_{1}}^{q_{2}} \frac{\Gamma\left(\xi+b+\frac{p}{\delta}\right)}{\Gamma\left(\xi+c+\frac{p}{\delta}\right)}$ are both rational functions if and only if $b-c$ is an integer, i.e., $\prod_{p=0}^{r_{1}} \frac{\Gamma\left(\xi+c+\frac{k}{\delta}+\frac{p}{\delta}\right) \Gamma\left(\xi+b+\frac{p}{\delta}\right)}{\Gamma\left(\xi+c+\frac{p}{\delta}\right) \Gamma\left(\xi+b+\frac{k}{\delta}+\frac{p}{\delta}\right)}$ is a rational function if and only if $b-c$ is an integer.

Moreover, if $a=c$, i.e., $k=\delta$, then $b=\frac{m}{2 \delta}$ and by (13) we get

$$
\begin{aligned}
\widehat{\psi}_{\alpha, \delta}(z) & =\prod_{p=0}^{\alpha} \frac{\Gamma\left(z / 2 \delta+b+\frac{p}{\delta}\right)}{\Gamma\left(z / 2 \delta+d+\frac{p}{\delta}\right)}=\prod_{p=0}^{\alpha} \frac{(2 \delta)^{\alpha+1}}{(z+2 b \delta+2 p)} \\
& =\frac{2 \delta^{\alpha+1}}{(\alpha+1) !}\left[\sum_{p=0}^{\alpha} C_{\alpha}^{p}(-1)^{p} \frac{1}{z+2 b \delta+2 p}\right] \\
& =\int_{0}^{1} \psi_{\delta}(r)\left(1-r^{2}\right)^{\alpha} r^{z-1} d r=\sum_{p=0}^{\alpha} C_{\alpha}^{p}(-1)^{p} \int_{0}^{1} \psi_{\delta}(r) r^{z+2 p-1} d r
\end{aligned}
$$

the uniqueness of the Mellin transform implies $\psi_{\delta}(r)=\frac{2 \delta^{\alpha+1}}{(\alpha+1) !} r^{2 b \delta}=\frac{2 \delta^{\alpha+1}}{(\alpha+1) !} r^{m}$.

If $a=c-1$, i.e., $k=0, a=0$, by (13) $b=d$ and we get

$$
\begin{aligned}
\widehat{\psi}_{\alpha, 0}(z) & =\prod_{p=0}^{\alpha} \frac{\Gamma\left(z / 2 \delta+\frac{p}{\delta}\right)}{\Gamma\left(z / 2 \delta+\frac{p}{\delta}\right)}=\prod_{p=0}^{\alpha} \frac{(2 \delta)^{\alpha+1}}{(z+2 p)}=\frac{2 \delta^{\alpha+1}}{(\alpha+1) !}\left[\sum_{p=0}^{\alpha} C_{\alpha}^{p}(-1)^{p} \frac{1}{z+2 p}\right] \\
& =\int_{0}^{1} \psi_{0}(r)\left(1-r^{2}\right)^{\alpha} r^{z-1} d r=\sum_{p=0}^{\alpha} C_{\alpha}^{p}(-1)^{p} \int_{0}^{1} \psi_{0}(r) r^{z+2 p-1} d r
\end{aligned}
$$

the uniqueness of the Mellin transform implies $\psi_{0}(r)=\frac{2 \delta^{\alpha+1}}{(\alpha+1) !}$. 


\section{Hyponormality of Toeplitz operators}

Now we start to discuss the hyponormality of Toeplitz operators on the weighted Bergman space $A_{\alpha}^{2}$, where $\alpha>-1$.

Theorem 3.1. Let $\varphi\left(r e^{i \theta}\right)=e^{i \delta \theta} \varphi_{0}(r) \in L^{\infty}(\mathbb{D})$, where $\delta \in \mathbb{Z}, \varphi_{0}(r) \in \mathscr{R}_{\alpha}$. Then $T_{\varphi}$ is hyponormal if and only if one of the following conditions is satisfied:

(1) $\delta<0$ and $\varphi_{0}=0$;

(2) $\delta=0$;

(3) $\delta>0$ and for each $n \geq \delta$,

$$
\left|\widehat{\varphi}_{\alpha, 0}(2 n+\delta+2)\right| \geq \sqrt{\frac{\Gamma(n-\delta+2+\alpha)(n+\delta) !}{\Gamma(n+\delta+2+\alpha)(n-\delta) !}}\left|\widehat{\varphi}_{\alpha, 0}(2 n-\delta+2)\right| .
$$

Proof. For any integer $n \in \mathbb{N}_{0}$, we have

$$
T_{\varphi} z^{n}=\left\{\begin{array}{cc}
\frac{(2 n+2 \delta+2) \Gamma(n+\delta+2+\alpha)}{(n+\delta+1) ! \Gamma(1+\alpha)} \widehat{\varphi}_{\alpha, 0}(2 n+\delta+2) z^{n+\delta}, & n+\delta \geq 0 \\
0, & n+\delta<0
\end{array}\right.
$$

and

$$
T_{\bar{\varphi}} z^{n}=\left\{\begin{array}{cc}
\frac{(2 n-2 \delta+2) \Gamma(n-\delta+2+\alpha)}{(n-\delta+1) ! \Gamma(1+\alpha)} \widehat{\varphi}_{\alpha, 0}(2 n-\delta+2) z^{n-\delta}, & n-\delta \geq 0 \\
0, & n-\delta<0
\end{array}\right.
$$

where $\widehat{\varphi}_{\alpha, 0}(z)=\int_{0}^{1} \varphi_{0}(r) r^{z-1}\left(1-r^{2}\right)^{\alpha} d r$ is the Mellin transform of the function $\varphi_{0}(r)\left(1-r^{2}\right)^{\alpha}$.

For each $f \in A_{\alpha}^{2}$ with $f(z)=\sum_{n=0}^{\infty} a_{n} z^{n}$, we get

$$
T_{\varphi} f=\sum_{n=0}^{\infty} a_{n} \frac{(2 n+2 \delta+2)^{+} \Gamma(n+\delta+2+\alpha)}{(n+\delta+1) ! \Gamma(1+\alpha)} \widehat{\varphi}_{\alpha, 0}(2 n+\delta+2) z^{n+\delta}
$$

and

$$
T_{\bar{\varphi}} f=\sum_{n=0}^{\infty} a_{n} \frac{(2 n-2 \delta+2)^{+} \Gamma(n-\delta+2+\alpha)}{(n-\delta+1) ! \Gamma(1+\alpha)} \widehat{\varphi}_{\alpha, 0}(2 n-\delta+2) z^{n-\delta},
$$

where $(2 n+2 \delta+2)^{+}=\max \{2 n+2 \delta+2,0\},(2 n-2 \delta+2)^{+}=\max \{2 n-2 \delta+2,0\}$. Note that

$$
\begin{aligned}
& \left(T_{\varphi} f, T_{\varphi} f\right) \\
= & \sum_{n=0}^{\infty}\left|a_{n}\right|^{2}\left[\frac{(2 n+2 \delta+2)+\Gamma(n+\delta+2+\alpha)}{(n+\delta+1) ! \Gamma(1+\alpha)}\right]^{2}\left|\widehat{\varphi}_{\alpha, 0}(2 n+\delta+2)\right|^{2}\left(z^{n+\delta}, z^{n+\delta}\right)
\end{aligned}
$$

and

$$
\begin{aligned}
& \left(T_{\bar{\varphi}} f, T_{\bar{\varphi}} f\right) \\
= & \sum_{n=0}^{\infty}\left|a_{n}\right|^{2}\left[\frac{(2 n-2 \delta+2)^{+} \Gamma(n-\delta+2+\alpha)}{(n-\delta+1) ! \Gamma(1+\alpha)}\right]^{2}\left|\widehat{\varphi}_{\alpha, 0}(2 n-\delta+2)\right|^{2}\left(z^{n-\delta}, z^{n-\delta}\right) .
\end{aligned}
$$


Suppose $\delta<0$. By (18) and (19) we have

$$
\begin{aligned}
& T_{\varphi} z^{n}=0, \\
& T_{\bar{\varphi}} z^{n}=\frac{(2 n-2 \delta+2) \Gamma(n-\delta+2+\alpha)}{(n-\delta+1) ! \Gamma(1+\alpha)} \widehat{\varphi}_{\alpha, 0}(2 n-\delta+2) z^{n-\delta}
\end{aligned}
$$

for $0 \leq n \leq-\delta-1$. If $T_{\varphi}$ is hyponormal, we get

$$
\widehat{\varphi}_{\alpha, 0}(2 n-\delta+2)=0 \quad \text { for } 0 \leq n \leq-\delta-1,
$$

i.e.,

$$
\widehat{\varphi}_{\alpha, 0}(p)=0 \quad \text { for any } p \in M_{0}=\{2(n+1)-\delta: 0 \leq n \leq-\delta-1, n \in \mathbb{Z}\},
$$

thus

$$
\begin{aligned}
& \left(T_{\varphi} f, T_{\varphi} f\right)-\left(T_{\bar{\varphi}} f, T_{\bar{\varphi}} f\right) \\
= & \sum_{n=-\delta}^{\infty} 4(\alpha+1)\left|a_{n}\right|^{2}\left[\frac{\Gamma(n+\delta+2+\alpha)}{(n+\delta) ! \Gamma(1+\alpha)}\left|\widehat{\varphi}_{\alpha, 0}(2 n+\delta+2)\right|^{2}\right. \\
& \left.-\frac{\Gamma(n-\delta+2+\alpha)}{(n-\delta) ! \Gamma(1+\alpha)}\left|\widehat{\varphi}_{\alpha, 0}(2 n-\delta+2)\right|^{2}\right] .
\end{aligned}
$$

Since $2 n+\delta+2=2(n+1+\delta)-\delta$, we have $2 n+\delta+2 \in M_{0}$ and $\widehat{\varphi}_{\alpha, 0}(2 n+\delta+2)=0$ for $-\delta \leq n \leq-2 \delta-1$, then by the hyponormality of $T_{\varphi}$ we get

$$
\widehat{\varphi}_{\alpha, 0}(2 n-\delta+2)=0 \quad \text { for }-\delta \leq n \leq-2 \delta-1,
$$

so we have

$$
\widehat{\varphi}_{\alpha, 0}(p)=0 \quad \text { for any } p \in M_{1}=\{2(n+1)-\delta: 0 \leq n \leq-2 \delta-1, n \in \mathbb{Z}\} \text {. }
$$

By the induction we could get $\widehat{\varphi}_{\alpha, 0}(p)=0$, where $p \in M_{\infty}=\{2(n+1)-\delta$ : $\left.n \in \mathbb{N}_{0}\right\}$, then by Lemma 3.1 in [18] we get $\varphi_{0}=0$. And it is clear that the converse holds.

Suppose $\delta=0$, it is evident that $\left(T_{\varphi} f, T_{\varphi} f\right)-\left(T_{\bar{\varphi}} f, T_{\bar{\varphi}} f\right) \geq 0$.

Suppose $\delta>0$, then

$$
\begin{aligned}
& \left(T_{\varphi} f, T_{\varphi} f\right)-\left(T_{\bar{\varphi}} f, T_{\bar{\varphi}} f\right) \\
= & \sum_{n=0}^{\delta-1} 4(\alpha+1)\left|a_{n}\right|^{2} \frac{\Gamma(n+\delta+2+\alpha)}{(n+\delta) ! \Gamma(1+\alpha)}\left|\widehat{\varphi}_{\alpha, 0}(2 n+\delta+2)\right|^{2} \\
& +\sum_{n=\delta}^{\infty} 4(\alpha+1)\left|a_{n}\right|^{2}\left[\frac{\Gamma(n+\delta+2+\alpha)}{(n+\delta) ! \Gamma(1+\alpha)}\left|\widehat{\varphi}_{\alpha, 0}(2 n+\delta+2)\right|^{2}\right. \\
& \left.-\frac{\Gamma(n-\delta+2+\alpha)}{(n-\delta) ! \Gamma(1+\alpha)}\left|\widehat{\varphi}_{\alpha, 0}(2 n-\delta+2)\right|^{2}\right] \geq 0
\end{aligned}
$$


if and only if

$$
\begin{aligned}
& \frac{\Gamma(n+\delta+2+\alpha)}{(n+\delta) ! \Gamma(1+\alpha)}\left|\widehat{\varphi}_{\alpha, 0}(2 n+\delta+2)\right|^{2}-\frac{\Gamma(n-\delta+2+\alpha)}{(n-\delta) ! \Gamma(1+\alpha)}\left|\widehat{\varphi}_{\alpha, 0}(2 n-\delta+2)\right|^{2} \\
& \geq 0 \quad \text { for } n \geq \delta
\end{aligned}
$$

if and only if

$$
\begin{aligned}
& \left|\widehat{\varphi}_{\alpha, 0}(2 n+\delta+2)\right| \\
\geq & \sqrt{\frac{\Gamma(n-\delta+2+\alpha)(n+\delta) !}{\Gamma(n+\delta+2+\alpha)(n-\delta) !}}\left|\widehat{\varphi}_{\alpha, 0}(2 n-\delta+2)\right| \quad \text { for } n \geq \delta .
\end{aligned}
$$

According to the above theorem we could identify whether a Toeplitz operator with the symbol as in the theorem is hyponormal. Now we give examples as follows:

Example 3.2. Let $\varphi_{1}\left(r e^{i \theta}\right)=e^{i \delta \theta} \varphi_{0}(r)=e^{i \delta \theta} H(\omega-r) \frac{1}{\left(1-r^{2}\right)^{\alpha}}$ and $\varphi_{2}\left(r e^{i \theta}\right)=$ $e^{i \delta \theta} r^{3 \delta}$, where $\delta>0, \omega=\frac{1}{2+\alpha}$ and $H$ is defined as follows:

$$
H(\omega-r)= \begin{cases}0, & \text { if } \omega<r<1 \\ \frac{1}{2}, & \text { if } r=\omega \\ 1, & \text { if } 0<r<\omega\end{cases}
$$

Then $T_{\varphi_{1}}$ is not hyponormal and $T_{\varphi_{2}}$ is hyponormal.

Proof. It is evident that $\varphi_{1} \in L^{\infty}(\mathbb{D})$ and $\varphi_{0}(r)=H(\omega-r) \frac{1}{\left(1-r^{2}\right)^{\alpha}}$. So $\widehat{\varphi}_{\alpha, 0}(z)=\frac{\omega^{z}}{z}$, and $\widehat{\varphi}_{\alpha, 0}(2 n+\delta+2)=\frac{\omega^{2 n+\delta+2}}{2 n+\delta+2}=\widehat{\varphi}_{\alpha, 0}(2 n-\delta+2) \frac{\omega^{2 \delta}(2 n-\delta+2)}{2 n+\delta+2}$. In particular, $\widehat{\varphi}_{\alpha, 0}(2 \delta+\delta+2)=\frac{\omega^{2 \delta+\delta+2}}{2 \delta+\delta+2}=\widehat{\varphi}_{\alpha, 0}(2 \delta-\delta+2) \frac{\omega^{2 \delta}(2 \delta-\delta+2)}{2 \delta+\delta+2}<$ $\sqrt{\frac{\Gamma(2+\alpha)(2 \delta) !}{\Gamma(2 \delta+2+\alpha)}} \widehat{\varphi}_{\alpha, 0}(2 \delta-\delta+2)$, since the function $f(x)=\frac{x}{x+u}$ is strictly monotone increasing on $(-u,+\infty)$ when $u>0$ and $0<f(x)<1$. Hence we obtain that $T_{\varphi_{1}}$ is not hyponormal.

Similarly it is easy to identify that $T_{\varphi_{2}}$ is hyponormal.

Theorem 3.3. Let $\varphi\left(r e^{i \theta}\right)=\sum_{k=-\infty}^{0} e^{i k \theta} \varphi_{k}(r) \in L^{\infty}(\mathbb{D})$. Then $T_{\varphi}$ is hyponormal if and only if $\varphi\left(r e^{i \theta}\right)=\varphi_{0}(r)$.

Proof. For any integer $n \in \mathbb{N}_{0}$,

$$
\begin{aligned}
& T_{\varphi} z^{n}=\sum_{k=-n}^{0} \frac{2 \Gamma(n+k+2+\alpha)}{(n+k) ! \Gamma(1+\alpha)} \widehat{\varphi}_{\alpha, k}(2 n+k+2) z^{n+k}, \\
& T_{\bar{\varphi}} z^{n}=\sum_{k=-\infty}^{0} \frac{2 \Gamma(n-k+2+\alpha)}{(n-k) ! \Gamma(1+\alpha)} \widehat{\varphi}_{\alpha, k}(2 n-k+2) z^{n-k},
\end{aligned}
$$


where $\widehat{\varphi}_{\alpha, k}(z)=\int_{0}^{1} \varphi_{k}(r) r^{z-1}\left(1-r^{2}\right)^{\alpha} d r$ is the Mellin transform of the function $\varphi_{k}(r)\left(1-r^{2}\right)^{\alpha}$. Thus

$$
\begin{aligned}
& \left(T_{\varphi} z^{n}, T_{\varphi} z^{n}\right)=4(\alpha+1) \sum_{k=-n}^{0} \frac{\Gamma(n+k+2+\alpha)}{(n+k) ! \Gamma(1+\alpha)}\left|\widehat{\varphi}_{\alpha, k}(2 n+k+2)\right|^{2}, \\
& \left(T_{\bar{\varphi}} z^{n}, T_{\bar{\varphi}} z^{n}\right)=4(\alpha+1) \sum_{k=-\infty}^{0} \frac{\Gamma(n-k+2+\alpha)}{(n-k) ! \Gamma(1+\alpha)}\left|\widehat{\varphi}_{\alpha, k}(2 n-k+2)\right|^{2} .
\end{aligned}
$$

Now we will show that if $T_{\varphi}$ is hyponormal, then $\widehat{\varphi}_{\alpha, k}(2 n+2-k)=0$ for any $n \in \mathbb{N}_{0}$, where $k=-1,-2, \ldots$.

Suppose $T_{\varphi}$ is hyponormal and for simplicity let

$$
\begin{aligned}
& \begin{array}{l}
A_{n}=\frac{\Gamma(1+\alpha)}{4(\alpha+1)}\left(T_{\varphi} z^{n}, T_{\varphi} z^{n}\right)=\sum_{k=-n}^{0} \frac{\Gamma(n+k+2+\alpha)}{(n+k) !}\left|\widehat{\varphi}_{\alpha, k}(2 n+k+2)\right|^{2}, \\
B_{n}=\frac{\Gamma(1+\alpha)}{4(\alpha+1)}\left(T_{\bar{\varphi}} z^{n}, T_{\bar{\varphi}} z^{n}\right)=\sum_{k=-\infty}^{0} \frac{\Gamma(n-k+2+\alpha)}{(n-k) !}\left|\widehat{\varphi}_{\alpha, k}(2 n-k+2)\right|^{2} .
\end{array} \\
& \text { If } n=0 \text {, we have }
\end{aligned}
$$

$$
\begin{aligned}
A_{0} & =\Gamma(2+\alpha)\left|\widehat{\varphi}_{\alpha, 0}(2)\right|^{2}, \\
B_{0} & =\sum_{k=-\infty}^{0} \frac{\Gamma(2-k+\alpha)}{(-k) !}\left|\widehat{\varphi}_{\alpha, k}(2-k)\right|^{2} \\
& =\Gamma(2+\alpha)\left|\widehat{\varphi}_{\alpha, 0}(2)\right|^{2}+\sum_{k=-\infty}^{-1} \frac{\Gamma(2-k+\alpha)}{(-k) !}\left|\widehat{\varphi}_{\alpha, k}(2-k)\right|^{2} .
\end{aligned}
$$

According to $A_{0} \geq B_{0}$, we can obtain

$$
\widehat{\varphi}_{\alpha, k}(2-k)=0, \quad \text { where } k=-1,-2, \ldots
$$

If $n=1$, we have

$$
\begin{aligned}
A_{1} & =\sum_{k=-1}^{0} \frac{\Gamma(3+k+\alpha)}{(1+k) !}\left|\widehat{\varphi}_{\alpha, k}(4+k)\right|^{2} \\
& =\Gamma(3+\alpha)\left|\widehat{\varphi}_{\alpha, 0}(4)\right|^{2}+\Gamma(2+\alpha)\left|\widehat{\varphi}_{\alpha,-1}(3)\right|^{2}, \\
B_{1} & =\sum_{k=-\infty}^{0} \frac{\Gamma(3-k+\alpha)}{(1-k) !}\left|\widehat{\varphi}_{\alpha, k}(4-k)\right|^{2} \\
& =\Gamma(3+\alpha)\left|\widehat{\varphi}_{\alpha, 0}(4)\right|^{2}+\sum_{k=-\infty}^{-1} \frac{\Gamma(3-k+\alpha)}{(1-k) !}\left|\widehat{\varphi}_{\alpha, k}(4-k)\right|^{2} .
\end{aligned}
$$

Then by $A_{1} \geq B_{1}$ and the equation (20) we can obtain

$$
\widehat{\varphi}_{\alpha, k}(4-k)=0, \text { where } k=-1,-2, \ldots
$$

Now suppose that the conclusion holds when $0 \leq n \leq M_{0}$, i.e.,

$$
\widehat{\varphi}_{\alpha, k}(2 n+2-k)=0 \text { for } 0 \leq n \leq M_{0} \text {, where } k=-1,-2, \ldots
$$


Then if $n=M_{0}+1$, we have

$$
\begin{aligned}
A_{M_{0}+1}= & \sum_{k=-\left(M_{0}+1\right)}^{0} \frac{\Gamma\left(M_{0}+3+k+\alpha\right)}{\left(M_{0}+1+k\right) !}\left|\widehat{\varphi}_{\alpha, k}\left(2 M_{0}+4+k\right)\right|^{2} \\
= & \frac{\Gamma\left(M_{0}+3+\alpha\right)}{\left(M_{0}+1\right) !}\left|\widehat{\varphi}_{\alpha, 0}\left(2 M_{0}+4\right)\right|^{2} \\
& +\sum_{k=-\left(M_{0}+1\right)}^{-1} \frac{\Gamma\left(M_{0}+3+k+\alpha\right)}{\left(M_{0}+1+k\right) !}\left|\widehat{\varphi}_{\alpha, k}\left(2 M_{0}+4+k\right)\right|^{2}, \\
B_{M_{0}+1}= & \frac{\Gamma\left(M_{0}+3+\alpha\right)}{\left(M_{0}+1\right) !}\left|\widehat{\varphi}_{\alpha, 0}\left(2 M_{0}+4\right)\right|^{2} \\
& +\sum_{k=-\infty}^{-1} \frac{\Gamma\left(M_{0}+3-k+\alpha\right)}{\left(M_{0}+1-k\right) !}\left|\widehat{\varphi}_{\alpha, k}\left(2 M_{0}+4-k\right)\right|^{2} .
\end{aligned}
$$

Observe that for each integer $k$ with $-1 \leq k \leq-\left(M_{0}+1\right)$, we have $\left(2 M_{0}+2-\right.$ $k)-\left(2 M_{0}+4+k\right)=-2 k-2 \geq 0$ and $0 \leq 2 M_{0}+2+2 k \leq 2 M_{0}$ (which implies $0 \leq M_{0}+1+k \leq M_{0}$ ), then there exists an integer $n_{k}$ with $0 \leq n_{k} \leq M_{0}$ such that $2 M_{0}+4+\bar{k}=2 n_{k}+2-k$. Thus we have $\widehat{\varphi}_{\alpha, k}\left(2 M_{0}+4+k\right)=0$, where $k \in \mathbb{Z}$ and $-1 \leq k \leq-\left(M_{0}+1\right)$. Then by the hypothesis and $A_{M_{0}+1} \geq B_{M_{0}+1}$ we can get

$$
\widehat{\varphi}_{\alpha, k}\left(2\left(M_{0}+1\right)+2-k\right)=0, \quad \text { where } k=-1,-2, \ldots
$$

Hence by the induction and the equations (20), (21), (22), and (23) we obtain that if $T_{\varphi}$ is hyponormal, then for any $n \in \mathbb{N}_{0} \widehat{\varphi}_{\alpha, k}(2 n+2-k)=0$, where $k=-1,-2, \ldots$.

Fix $k$. Then by Lemma 3.1 in [18] we get $\varphi_{k}=0$, where $k=-1,-2, \ldots$, so $\varphi\left(r e^{i \theta}\right)=\varphi_{0}(r)$.

It is clear that the converse is true.

Example 3.4. By Theorem 3.3 we can easily get that $T_{\varphi}$ is not hyponormal when $\varphi\left(r e^{i \theta}\right)=e^{-i \delta \theta} r^{m}$, where $\delta, m \in \mathbb{N}$.

Theorem 3.5. Let $\varphi\left(r e^{i \theta}\right)=\sum_{k=-\infty}^{\infty} e^{i k \theta} \varphi_{k}(r) \in L^{\infty}(\mathbb{D})$ for any $\left\{a_{n}\right\}_{n=0}^{\infty}$ with $\sum_{n=0}^{\infty} \frac{n ! \Gamma(2+\alpha)}{\Gamma(n+2+\alpha)}\left|a_{n}\right|^{2}<+\infty$. If $\left\{\varphi_{k}\right\}_{k=-\infty}^{\infty}$ satisfy the following inequality:

$$
\left|\sum_{k=0}^{\infty} a_{k} \widehat{\varphi}_{\alpha, p-k}(p+k+2)\right| \geq\left|\sum_{k=0}^{\infty} a_{k} \widehat{\varphi}_{\alpha, k-p}(p+k+2)\right|
$$

for any $p \geq 0$, then $T_{\varphi}$ is hyponormal.

Proof. For any $f \in A_{\alpha}^{2}$ with $f=\sum_{k=0}^{\infty} a_{k} z^{k}$, we have

$$
T_{\varphi} f=\sum_{k=0}^{\infty} a_{k} T_{\varphi} z^{k}
$$




$$
\begin{aligned}
& =\sum_{k=0}^{\infty} a_{k} \sum_{n=-k}^{\infty} \frac{(2 n+2 k+2) \Gamma(n+k+2+\alpha)}{(n+k+1) ! \Gamma(1+\alpha)} \widehat{\varphi}_{\alpha, n}(2 k+n+2) z^{n+k} \\
& =\sum_{k=0}^{\infty} a_{k} \sum_{p=0}^{\infty} \frac{(2 p+2) \Gamma(p+2+\alpha)}{(p+1) ! \Gamma(1+\alpha)} \widehat{\varphi}_{\alpha, p-k}(p+k+2) z^{p} \\
& =\sum_{p=0}^{\infty} \frac{(2 p+2) \Gamma(p+2+\alpha)}{(p+1) ! \Gamma(1+\alpha)}\left[\sum_{k=0}^{\infty} a_{k} \widehat{\varphi}_{\alpha, p-k}(p+k+2)\right] z^{p}
\end{aligned}
$$

and

$$
\begin{aligned}
T_{\bar{\varphi}} f & =\sum_{k=0}^{\infty} a_{k} T_{\bar{\varphi}} z^{k} \\
& =\sum_{k=0}^{\infty} a_{k} \sum_{n=-\infty}^{k} \frac{(2 k-2 n+2) \Gamma(k-n+2+\alpha)}{(k-n+1) ! \Gamma(1+\alpha)} \widehat{\varphi}_{\alpha, n}(2 k-n+2) z^{k-n} \\
& =\sum_{k=0}^{\infty} a_{k} \sum_{p=0}^{\infty} \frac{(2 p+2) \Gamma(p+2+\alpha)}{(p+1) ! \Gamma(1+\alpha)} \widehat{\varphi}_{\alpha, k-p}(p+k+2) z^{p} \\
& =\sum_{p=0}^{\infty} \frac{(2 p+2) \Gamma(p+2+\alpha)}{(p+1) ! \Gamma(1+\alpha)}\left[\sum_{k=0}^{\infty} a_{k} \widehat{\varphi}_{\alpha, k-p}(p+k+2)\right] z^{p} .
\end{aligned}
$$

Then

$$
\begin{aligned}
& \left(T_{\varphi} f, T_{\varphi} f\right)=\sum_{p=0}^{\infty} \frac{4(\alpha+1) \Gamma(p+2+\alpha)}{p ! \Gamma(1+\alpha)}\left|\sum_{k=0}^{\infty} a_{k} \widehat{\varphi}_{\alpha, p-k}(p+k+2)\right|^{2}, \\
& \left(T_{\bar{\varphi}} f, T_{\bar{\varphi}} f\right)=\sum_{p=0}^{\infty} \frac{4(\alpha+1) \Gamma(p+2+\alpha)}{p ! \Gamma(1+\alpha)}\left|\sum_{k=0}^{\infty} a_{k} \widehat{\varphi}_{\alpha, k-p}(p+k+2)\right|^{2},
\end{aligned}
$$

and

$$
\begin{aligned}
& \left(T_{\varphi} f, T_{\varphi} f\right)-\left(T_{\bar{\varphi}} f, T_{\bar{\varphi}} f\right) \\
= & \sum_{p=0}^{\infty} \frac{4(\alpha+1) \Gamma(p+2+\alpha)}{p ! \Gamma(1+\alpha)}\left[\left|\sum_{k=0}^{\infty} a_{k} \widehat{\varphi}_{\alpha, p-k}(p+k+2)\right|\left|\sum_{k=0}^{\infty} a_{k} \widehat{\varphi}_{\alpha, k-p}(p+k+2)\right|^{2}\right] .
\end{aligned}
$$

If the inequality (24) is true, then we have

$$
\left(T_{\varphi} f, T_{\varphi} f\right)-\left(T_{\bar{\varphi}} f, T_{\bar{\varphi}} f\right) \geq 0,
$$

that is, $T_{\varphi}$ is hyponormal.

\section{References}

[1] S. Axler and Z̆. Cučković, Commuting Toeplitz operators with harmonic symbols, Integral Equations Operator Theory 14 (1991), no. 1, 1-12.

[2] S. Axler, Z. Cučković, and N. V. Rao, Commutants of analytic Toeplitz operators on the Bergman space, Proc. Amer. Math. Soc. 128 (2000), no. 7, 1951-1953. 
[3] A. Brown and P. R. Halmos, Algebraic properties of Toeplitz operators, J. Reine Angew. Math. 213 (1963), 89-102.

[4] B. R. Choe, H. Koo, and Y. J. Lee, Commuting Toeplitz operators on the polydisk, Trans. Amer. Math. Soc. 356 (2004), no. 5, 1727-1749.

[5] J. B. Conway, Functions of One Complex Variable, Second edition. Graduate Texts in Mathematics, 11. Springer-Verlag, New York-Berlin, 1978.

[6] C. C. Cowen, Hyponormal and subnormal Toeplitz operators, Surveys of some recent results in operator theory, Vol. I, 155-167, Pitman Res. Notes Math. Ser., 171, Longman Sci. Tech., Harlow, 1988.

[7] _ Hyponormality of Toeplitz operators, Proc. Amer. Math. Soc. 103 (1988), no. 3, 809-812.

[8] Z̆eljko Cučcković and N. V. Rao, Mellin transform, monomial symbols, and commuting Toeplitz operators, J. Funct. Anal. 154 (1998), no. 1, 195-214.

[9] R. E. Curto and W. Y. Lee, Joint hyponormality of Toeplitz pairs, Mem. Amer. Math. Soc. 150 (2001), no. 712, x+65 pp.

[10] P. Fan, Remarks on hyponormal trigonometric Toeplitz operators, Rocky Mountain J. Math. 13 (1983), no. 3, 489-493.

[11] D. R. Farenick and W. Y. Lee, Hyponormality and spectra of Toeplitz operators, Trans. Amer. Math. Soc. 348 (1996), no. 10, 4153-4174.

[12] C. X. Gu, A generalization of Cowen's characterization of hyponormal Toeplitz operators, J. Funct. Anal. 124 (1994), no. 1, 135-148.

[13] - On a class of jointly hyponormal Toeplitz operators, Trans. Amer. Math. Soc. 354 (2002), no. 8, 3275-3298.

[14] I. S. Hwang, Hyponormal Toeplitz operators on the Bergman space, J. Korean Math. Soc. 42 (2005), no. 2, 387-403.

[15] I. S. Hwang, I. H. Kim, and W. Y. Lee, Hyponormality of Toeplitz operators with polynomial symbols, Math. Ann. 313 (1999), no. 2, 247-261.

[16] I. S. Hwang and W. Y. Lee, Hyponormality of trigonometric Toeplitz operators, Trans. Amer. Math. Soc. 354 (2002), no. 6, 2461-2474.

[17] Y. J. Lee, Pluriharmonic symbols of commuting Toeplitz type operators on the weighted Bergman spaces, Canad. Math. Bull. 41 (1998), no. 2, 129-136.

[18] I. Louhichi and E. Strouse and Elizabeth and L. Zakariasy, Products of Toeplitz operators on the Bergman space, Integral Equations Operator Theory 54 (2006), no. 4, 525-539.

[19] Y. F. Lu, Commuting of Toeplitz operators on the Bergman spaces of the bidisc, Bull. Austral. Math. Soc. 66 (2002), no. 2, 345-351.

[20] T. Nakazi and K. Takahashi, Hyponormal Toeplitz operators and extremal problems of Hardy spaces, Trans. Amer. Math. Soc. 338 (1993), no. 2, 753-767.

[21] H. Sadraoui, Hyponormality of Toeplitz operators and Composition operators, Thesis, Purdue University, 1992.

[22] D. Sarason, Generalized interpolation in $H^{\infty}$, Trans. Amer. math. Soc. 127 (1967), 179-203.

[23] K. Stroethof, Essentially commuting Toeplitz operators with harmonic symbols, Canad. J. Math. 45 (1993), no. 5, 1080-1093.

[24] E. T. Whittaker and G. N. Watson, A Course of Modern Analysis. An Introduction to the General Theory of Infinite Processes and of Analytic Functions with an Account of the Principal Transcendental Functions, Fourth edition. Reprinted Cambridge University Press, New York 1962.

[25] D. Zheng, Commuting Toeplitz operators with pluriharmonic symbols, Trans. Amer. Math. Soc. 350 (1998), no. 4, 1595-1618.

[26] K. H. Zhu, Hyponormal Toeplitz operators with polynomial symbols, Integral Equations Operator Theory 21 (1995), no. 3, 376-381. 
YUFENG LU

Department of Applied Mathematics

DALIAN UNIVERSITY OF TECHNOLOGY

Dalian 116024, Liaoning, P. R. China

E-mail address: lyfdlut@dlut.edu.cn; lyfdlut1@yahoo.com.cn

Chaomei LiU

Department of Applied Mathematics

DALIAN UNIVERSITY OF TECHNOLOGY

Dalian 116024, Liaoning, P. R. China

E-mail address: liuchaomei8092@126.com 